\title{
Codelivery of temozolomide and siRNA with polymeric nanocarrier for effective glioma treatment
}

This article was published in the following Dove Press journal: International Journal of Nanomedicine

\author{
Yuan Peng ${ }^{1,2}$ \\ Jinsheng Huang ${ }^{2}$ \\ Hong Xiao ${ }^{2}$ \\ Teng Wu' \\ Xintao Shuai ${ }^{1,2}$ \\ 'Center of Biomedical Engineering, \\ Zhongshan School of Medicine, \\ Sun Yat-Sen University, Guangzhou \\ 5 I 0080, China; ${ }^{2}$ Key Laboratory for \\ Polymeric Composite \& Functional \\ Materials of Ministry of Education, \\ Department of Polymer Science, \\ School of Materials Science and \\ Engineering, Sun Yat-Sen University, \\ Guangzhou 510275, China
}

Background: The treatment of glioma remains a challenge because conventional chemotherapy is often ineffective by drug resistance. Combinative therapy using chemotherapeutic agents and siRNA has demonstrated potential to improve anticancer outcome through a synergistic effect in various cancers. The current study aims to achieve better glioma treatment through a combinative therapy based on a folate-targeted nanocarrier carrying both temozolomide (TMZ) and anti-BCL-2 siRNA.

Methods: A polymeric micelle (TMZ-FaPEC@siRNA) incorporating TMZ and anti-BCL-2 siRNA was prepared based on folate-conjugated triblock copolymer (Fa-PEG-PEI-PCL, FaPEC) of poly( $\varepsilon$-caprolactone) (PCL), poly(ethylenimine) (PEI) and poly(ethylene glycol) (PEG). The physicochemical properties and drug release profile of TMZ-FaPEC@siRNA were tested. The Fa-targeted drug delivery and joint effect of siRNA and TMZ to induce glioma apoptosis and tumor growth inhibition were evaluated both in vitro and in vivo.

Results: In vitro cell study demonstrated that the nanocarrier effectively facilitates codelivery of siRNA and TMZ into C6 cells, resulting in a strong apoptotic response of cancer cells by silencing the antiapoptosis BCL-2 gene and activating the proapoptotic Bax gene simultaneously. In vivo study in rat bearing orthotropic glioma showed that tumor growth was inhibited and meanwhile animal survival was prolonged remarkably through intracranial injection of TMZ-FaPEC@siRNA.

Conclusion: Our results evidence the strong efficacy of the folate-targeted nanomedicine carrying TMZ and BCL-2 siRNA in treating glioma.

Keywords: polymeric nanocarrier, temozolomide, siRNA targeting BCL-2 gene, glioma treatment, drug resistance

\section{Introduction}

Glioma is a fatal malignancy in central nervous system (CNS) with a median survival period of only 14 months and a 5 -year survival rate $<10 \%$ after diagnosis. ${ }^{1,2}$ Current means for clinical glioma treatment include surgical resection, radiation, and chemotherapy. In particular, chemotherapy using temozolomide (TMZ) as the standard chemotherapeutic plan has shown ascertained effect to increase the survival rate of patients suffering from this disease. ${ }^{2-4}$ However, owing to the inherent and acquired resistance of glioma cells to TMZ as well as the blood-brain barrier (BBB) impeding the orally or intravenously administered drug to enter the brain, the effect of TMZ chemotherapy is greatly limited. ${ }^{5}$ Although the restriction of BBB for drug delivery may be overcome by local drug administration, drug resistance is still a big challenge for TMZ treatment to exert an ideal effect. 
Synthetic siRNA has been applied in the therapeutic studies of various diseases including cancer. ${ }^{6}$ As a major agent for gene therapy, siRNA exhibits not only high efficiency to induce target gene silencing but also enhanced safety compared to DNA as it only interferes with the posttranscriptional process. ${ }^{7,8}$ Previous studies have shown that upregulated expressions of antiapoptotic proteins may make cancer cells less sensitive to chemotherapy, which is known as a non-pump resistance. ${ }^{910}$ As a major antiapoptosis protein, BCL-2 impedes the mitochondrial release of apoptosis activating factor and cytochrome $\mathrm{c}$ initiating the caspase cascade. ${ }^{11}$ As BCL-2 is overexpressed in a variety of cancer types including glioma, ${ }^{12} \mathrm{BCL}-2$ silencing using siRNA is regarded as a promising approach to overcome chemoresistance and promote tumor apoptosis. ${ }^{13}$ In this context, a combinative glioma therapy using TMZ and BCL-2 siRNA may result in improved outcome. Nevertheless, the strategy relies on the development of an efficient codelivery system for the two different kinds of therapeutic agents. ${ }^{14}$

It is well known that free siRNA is prone to nuclease degradation in vivo and its cell uptake is usually hindered by negative charge. ${ }^{15}$ So far, a number of delivery systems including viral and nonviral vectors have been developed to facilitate siRNA therapy. Among the nonviral ones, cationic polymers have been widely studied because of their easy synthesis and good biocompatibility. ${ }^{16}$ In particular, poly(ethylenimine) (PEI) is acknowledged as the most promising one not only because it readily complexes siRNA but also because it facilitates the lyosomal escape of nanocarriers through proton sponge effect. ${ }^{17}$ However, the issue may become much more complicated in designing vectors for the codelivery purpose. A main reason is that two different types of therapeutic agents often possess very different physicochemical properties and thus require different structural materials to accommodate them.

Up to now, multifunctional nanoplatforms developed for the codelivery purpose mainly include polymeric, liposomal, and silica-based cationic nanoparticles. ${ }^{18}$ Surface PEGylation of nanoparticles was generally needed in order to improve the serum stability of nanoparticles and to shield the cationic layer for decreased cytotoxicity. Recently, we have achieved the codelivery of siRNA and chemotherapeutic agents in vitro using the folate-conjugated triblock copolymer of poly( $\varepsilon$-caprolactone) (PCL), PEI, and poly(ethylene glycol) (PEG) (Fa-PEG-PEI-PCL). ${ }^{19}$ Cell studies showed that the codelivered siRNA could effectively downregulate the expression of antiapoptosis genes to sensitize ovarian cancer cells to chemotherapeutic drug doxorubicin, ${ }^{19}$ which enlightened us to explore whether this concept may work as well in a TMZ-based chemotherapy of glioma, especially in the animal tumor model.

A polymeric micelle (TMZ-FaPEC@siRNA) incorporating TMZ and siRNA targeting the antiapoptosis BCL-2 gene was prepared, as shown in Figure 1. Through hydrophobic interaction, Fa-PEG-PEI-PCL self-assembled into micelle encapsulating TMZ in the hydrophobic core. Then, siRNA was complexed with the cationic micelle through electrostatic interaction. With folate as targeting molecule on the surface layer, the micelle was expected to be readily internalized into glioma cells, where folate receptor (FR) is significantly overexpressed as compared to normal cells. ${ }^{20}$ To explore the therapeutic potential of TMZ-FaPEC@siRNA, the codelivery efficiency, joint effect of BCL-2 silencing and TMZ to induce glioma apoptosis, and tumor growth inhibition were evaluated in glioma $\mathrm{C} 6$ cells and animal model. A direct injection of the drug-loaded micelle to the right striatum of animal was adopted in an animal study in consideration that BBB not only prevents drugs from entering the brain but also helps the sequestration of intracranially applied drug. ${ }^{21}$

\section{Materials and methods Materials}

$\alpha$-Allyl- $\varepsilon$-hydroxy-poly(ethylene glycol) (Allyl-PEG-OH, $\mathrm{Mn}=2.0 \mathrm{kDa})$, methoxy-poly(ethylene glycol) $(\mathrm{Mn}=2.0$ $\mathrm{kDa}$, N-hydroxysuccinimide, 1-dodecanol, N, $\mathrm{N}^{\prime}$ carbonyldiimidazole (CDI), folic acid (Fa), stannous (II) octoate, cysteamine hydrochloride, dicyclohexylcarbodiimide, and triethylamine were purchased from Sigma-Aldrich and used as obtained. TMZ was obtained from Zhejiang Pharmaceutical Co. Ltd (Zhejiang, China). $\varepsilon$-Caprolactone (Sigma-Aldrich) was dried with $\mathrm{CaH}_{2}$ and purified by vacuum distillation. The scramble siRNA (SCR) and two anti-BCL-2 siRNA duplexes with sequences GGAUGACUGAGUA CCUGAATT (dTdT) as passenger strand, UUCAGGU ACUCAGUCAUCCTT (dTdT) as guide strand were from Genpharm (Shanghai, China). Coumarin 6 and CCK8 cell proliferation assay kit were purchased from Sigma-Aldrich. Cy3-labeled siRNA was provided by Invitrogen Corporation (Carlsbad, CA, USA). The rat C6 glioma cell line overexpressing folate receptors was purchased from American Type Culture Collection. The fluorescent staining agent 4',6-diamidino-2-phenylindole was obtained from Roche, Germany. Terminal deoxynucleotidyl transferase dUTP nick end labeling (TUNEL) kit and enhanced chemiluminescence substrate were from Keygen biotech. Co. Ltd (Nanjing, China). Penicillin/streptomycin solution, Roswell Park Memorial Institute-1640 medium (RPMI-1640), and fetal bovine serum (FBS) were purchased from Gibco Life 


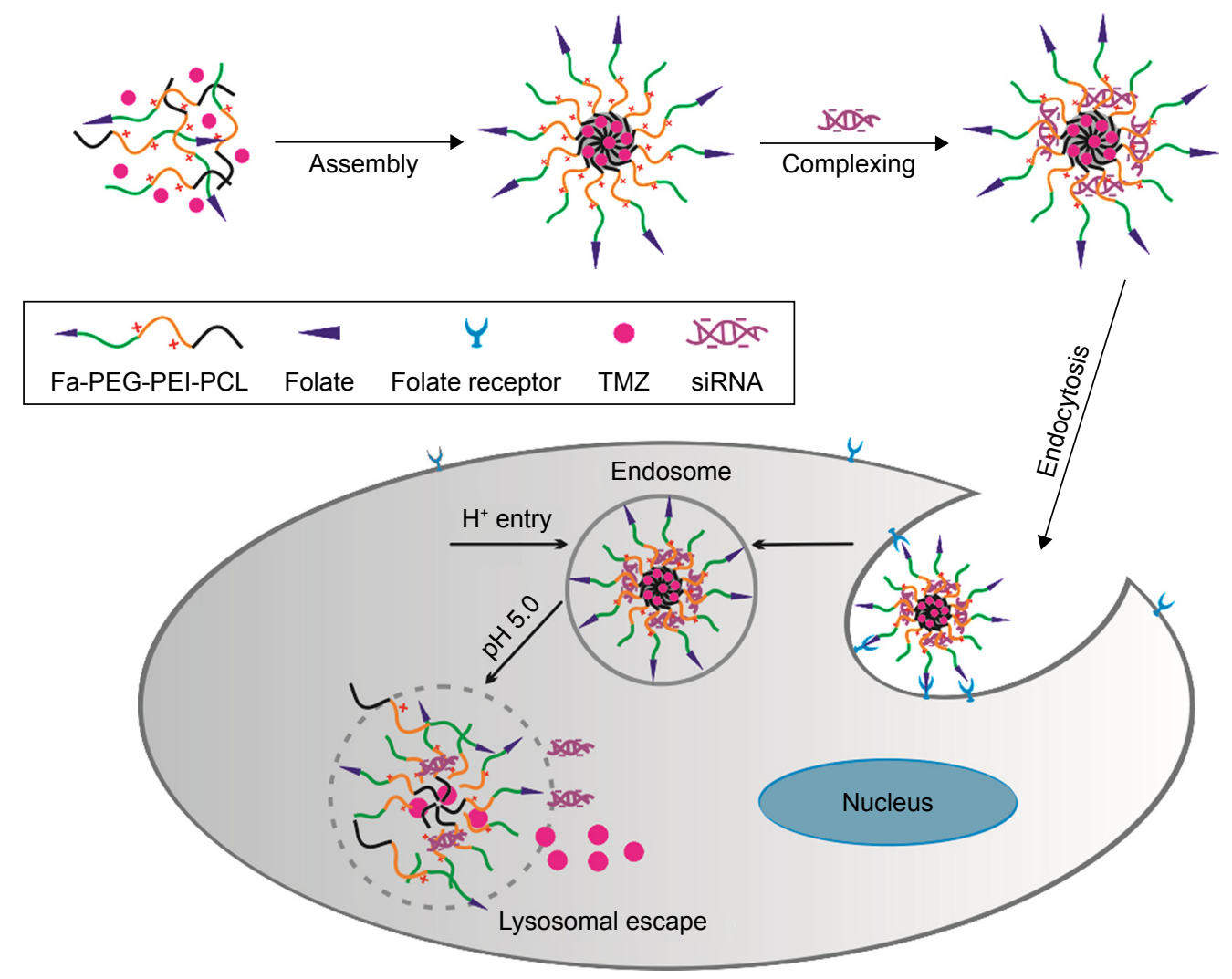

Figure I Schematic illustration of the preparation for TMZ-FaPEC@siRNA micelle and the release of TMZ and siRNA inside cancer cells. Abbreviations: Fa, folate; PCL, poly(E-caprolactone); PEG, poly(ethylene glycol); PEI, poly(ethylenimine); TMZ, temozolomide.

Technology (Carlsbad, CA, USA). Nonfat milk and bovine serum albumin (BSA) were provided by Shanghai Sangon Biotech (Shanghai, China). Annexin V-fluorescein isothiocyanate (FITC)/propidium iodide (PI) Apoptosis Detection Kit was supplied by Absin Biochemical Company. Reverse transcriptional polymerase chain reaction (PCR) kit was from Takara (Nojihigashi, Japan) and the qPCR kit was obtained from Roche Diagnostics. All other reagents having analytical or higher purity were used as received.

\section{Preparation of Fa-PEG-PEI-PCL}

Monohydroxyl-terminated polycaprolactone $(\mathrm{Mn}=2.3 \mathrm{kDa}$, estimated by proton nuclear magnetic resonance [ $\left.\left.{ }^{1} \mathrm{H} \mathrm{NMR}\right]\right)$ was synthesized and activated with CDI to obtain PCL-CDI. Fa-conjugated decorated poly(ethylene glycol)- $b$-branched poly(ethylene amine) (Fa-PEG-PEI) and methoxy-poly(ethylene glycol)- $b$-branched poly(ethylene amine) (mPEG-PEI) were synthesized as reported and then further reacted with PCL-CDI to form Fa-PEG-PEI-PCL, abbreviated as FaPEC. ${ }^{19}$

\section{Preparation of TMZ- and siRNA-coloaded micelle}

Preparation of TMZ-loaded FaPEC (TMZ-FaPEC) and blank FaPEC cationic micelles (FaPEC) is shown as outlined in Figure 1. Coumarin-6 (3-(2-benzothiazolyl)-N,Ndiethylumbelliferylamine), which emits green fluorescence under appropriate light stimulation, was used to substitute TMZ for tracking intracellular distribution of the micelle (Coum6-FaPEC). The micelle without folate modification was prepared through the same procedure except that mPEGPEI-PCL rather than Fa-PEG-PEI-PCL was used.

A predetermined amount of siRNA (eg, $0.02 \mathrm{mg}$ ) was mixed with a certain amount of TMZ-FaPEC micelle by gently pipetting for $1 \mathrm{~min}$. The solution was then incubated for $30 \mathrm{~min}$ at room temperature. By this means, various siRNA-loaded nanocomplexes (TMZ-FaPEC@siRNA and TMZ-PEC@siRNA) were prepared at different ratios of nitrogen in branched PEI to phosphorus in siRNA, which are generally referred to as $\mathrm{N} / \mathrm{P}$ ratios. The nanocomplexes without TMZ were prepared by the same procedure. Particle size, zeta potential, and drug-loading content were measured according to our previous report. ${ }^{19} \mathrm{TMZ}$ release profile was determined as follows: $4 \mathrm{~mL}$ sample solution of TMZFaPEC@SCR or TMZ-FaPEC@siRNA in PBS of pH 5.0 or pH 7.4 was transferred into a dialysis bag (molecular weight cutoff, $7 \mathrm{kDa}$ ). The dialysis bag was immersed in $8 \mathrm{~mL}$ PBS of the same $\mathrm{pH}$ in a beaker. The solution was then shaken at $37^{\circ} \mathrm{C}$. Four milliliters of solution outside the dialysis bag 
was taken out for high-performance liquid chromatography analysis and the same volume of fresh PBS was added into the beaker at a certain time interval. The amount of released drug was calculated based on a pre-established calibration curve, and the percentages of drug released from the micelle were plotted against drug release time. All experiments were conducted in triplicate.

\section{Agarose gel retardation assay}

The complexation of siRNA with TMZ-loaded micelle was analyzed by agarose gel electrophoresis. In detail, complexes of SCR $(1 \mu \mathrm{g})$ were prepared with $\mathrm{N} / \mathrm{P}$ ratios ranging from 0 to 12 and diluted to $2 \mu \mathrm{L}$ with $50 \%$ glycerol. Then, the samples were transferred to $1 \%$ agarose gels containing ethidium bromide $(0.5 \mu \mathrm{g} / \mathrm{mL})$ and ran with Tris-acetate buffer at $120 \mathrm{~V}$ for $15 \mathrm{~min}$. DNR Bio-Imaging System (NR Bio-Imaging System Ltd, Israel) was utilized to detect the retardation of siRNA motion.

\section{Cell culture and transfection}

Rat glioma C6 cells were cultured as previously reported. ${ }^{16}$ Cells were seeded in 24-well plates with $\sim 1 \times 10^{4}$ cells in each well. Subsequently they were incubated for $12 \mathrm{~h}$ in $1 \mathrm{~mL}$ of RPMI-1640 with 10\% FBS, $4 \mathrm{~h}$ in $1 \mathrm{~mL}$ of Fa-deficient and FBS-free RPMI-1640, and another $2 \mathrm{~h}$ after adding micelle to the medium at an siRNA concentration of $25 \mathrm{nM}$. For the free folate inhibition assay, cells were pretreated with folate ( $1 \mathrm{mg} / \mathrm{L})$ for $30 \mathrm{~min}$ before the targeting micelle was added to the culture medium. Intracellular distribution of the nanoparticles was observed with confocal laser scanning microscopy. Cell transfection efficiency was measured by flow cytometry.

\section{Cell viability assay}

CCK8 assay was conducted to assess the cytotoxicity of the nanocomplexes. C6 cells were seeded in 96-well plates at $1 \times 10^{3}$ cells per well and then incubated for $12 \mathrm{~h}$ in $100 \mu \mathrm{L}$ of RPMI-1640 containing 10\% FBS. They were then incubated for $96 \mathrm{~h}$ in the Fa-deficient and FBS-free medium containing the following samples: 1) PEG-PEI-PCL micelle carrying SCR (PEC@SCR); 2) Fa-PEG-PEI-PCL micelle carrying SCR (FaPEC@SCR); 3) Fa-targeted micelle loading TMZ and siRNA (TMZ-FaPEC@siRNA, TMZ-FaPEC@SCR); and 4) non-Fa-targeting micelle loading TMZ and siRNA (TMZ-PEC@siRNA, TMZ-PEC@SCR). siRNA concentration was $20 \mathrm{nM}$ in each well for all groups. The medium was then changed to $150 \mu \mathrm{L}$ serum-free growth medium and $100 \mu \mathrm{L} 1640$ medium containing $10 \mu \mathrm{L} \mathrm{CCK} 8$. After an additional $4 \mathrm{~h}$ incubation, the absorbance of formazan crystals was measured on Infinite F200 Multimode plate reader (Tecan,
Crailsheim, Germany) at $450 \mathrm{~nm}$ after $4 \mathrm{~h}$ incubation. All experiments were conducted in triplicate.

\section{Cell apoptosis}

The $\mathrm{C} 6$ cells were cultured overnight at $37^{\circ} \mathrm{C}$ at $1 \times 10^{5}$ cells per well in six-well plates. After $48 \mathrm{~h}$ incubation with different micelle formulations (TMZ-FaPEC@siRNA, TMZPEC@siRNA, TMZ-FaPEC@SCR, FaPEC@siRNA, and free TMZ), the cells were trypsinized, collected, and washed with PBS, and resuspended in $500 \mu \mathrm{L}$ binding buffer added with $5 \mu \mathrm{L}$ Annexin V-FITC as well as PI solution. After the cells were incubated for another $15 \mathrm{~min}$, cell apoptosis was evaluated by flow cytometry. The excitation and emission wavelengths for Annexin V-FITC were 488 and $530 \mathrm{~nm}$, and for probes PI were 536 and $617 \mathrm{~nm}$, respectively.

\section{Molecular biology assays in vitro}

Expressions of BCL-2 and Bax at mRNA and protein levels were evaluated by real-time PCR (RT-PCR) and Western blot assays as previously reported. ${ }^{19}$ Briefly, C6 cells were treated with RIPM lysis and extraction buffer (Beyotime, Shanghai, China) according to the manufacturer's protocol. The extracted total protein was quantified using BCA assay (ThermoFisher, Waltham, MA, USA). Thirty micrograms of protein separated through $12 \%$ sodium dodecyl sulfate polyacrylamide gel electrophoresis was electrotransferred onto the polyvinylidene difluoride membranes (Millipore, Billerica, MA, USA). The membranes were washed with TBST, blocked by $5 \%$ of skim milk, and incubated at $4^{\circ} \mathrm{C}$ for $12 \mathrm{~h}$ with rabbit antibodies against BCL-2 and Bax (1:1,000 dilution, Abcam, USA) as well as rabbit antibody against $\beta$-actin (1:1,000 dilution; Santa Cruz Biotechnology, Santa Cruz, CA, USA) as an internal standard for normalizing protein expressions. After $1 \mathrm{~h}$ incubation with goat anti-rabbit immunoglobulin G (IgG) horseradish peroxidase (1:3,000, Santa Cruz Biotechnology, Santa Cruz, CA, USA) to amplify the signal, the membranes were subjected to chemiluminescent detection.

\section{Rat C6 glioma model}

In vivo studies were carried out with male Sprague Dawley rats (250-280 g). Animal experiments were approved by the Institutional Animal Care and Use Committee of the Sun YatSen University. Animals received care following the National Laboratory Animal Management Regulations (China, 1988) and Guidance on the Treatment of Experimental Animals (China, 2006). The in situ glioma model was constructed by the stereotactic implantation of $\mathrm{C} 6$ cells according to literature. ${ }^{22}$ Briefly, C6 cells dispersed in $20 \mu \mathrm{L}$ serum-free 
RPIM-1640 $\left(2.5 \times 10^{4}\right.$ cells $\left./ \mu \mathrm{L}\right)$ were injected for $10 \mathrm{~min}$ into the right caudate nucleus of the ketamine-anesthetized rats. After slowly withdrawing the syringe, the burr hole was sealed off with bone wax.

\section{In vivo studies}

Five days after the implantation, the 40 rats were evenly divided into five groups and injected with PBS, TMZFaPEC@siRNA, TMZ-PEC@siRNA,TMZ-FaPEC@SCR, and FaPEC@siRNA, respectively. Treatments were given every four days at the doses of $120 \mu \mathrm{g} / \mathrm{kg} \mathrm{TMZ}$ and $1.6 \mu \mathrm{g} / \mathrm{kg}$ siRNA. The five injection points for each treatment were 4 , $4.5,5,5.5$, and $6 \mathrm{~mm}$ away from the dura, respectively, and the needle was kept still for 5 min before retraction. Twentyfive days after the first injection, Gd-DTPA (Bayer Schering Pharma AG, Berlin, Germany) was intravenously administered at a dose of $150 \mu \mathrm{L} / \mathrm{kg}$, and then the rats were subjected to magnetic resonance imaging (MRI) for the measurement of tumor volume. T1-weighted sequence was employed to acquire images as reported. ${ }^{19}$ The mean tumor volume $\left(\mathrm{mm}^{3}\right)$ was calculated as $\pi \times a \times b \times c / 2$, in which $\mathrm{a}, \mathrm{b}$, and c represented coronal long diameter, coronal short diameter, and sagittal long diameter, respectively.

\section{Histology and immunohistochemistry}

Tumors from the killed rats were fixed by $10 \%$ formalin in PBS for $24 \mathrm{~h}$. Hematoxylin and eosin (H\&E) staining was conducted to observe the histologic changes of the tissue, with at least five sections examined for each rat. Cell apoptosis was evaluated by TUNEL assay with an In Situ Cell Death Detection Kit (Roche, USA) following the manufacturer's protocol. The expression of BCL-2 was evaluated by immunohistochemistry study. Briefly, after deparaffinization, the sections were were treated with $10 \mathrm{mM}$ citrate buffer for $15 \mathrm{~min}$ at $90^{\circ} \mathrm{C}$ and $0.3 \%$ hydrogen peroxide in methanol for $30 \mathrm{~min}$ at $4{ }^{\circ} \mathrm{C}$. After being blocked by $2 \%$ BSA, $10 \%$ normal horse serum, and $0.5 \%$ Triton $\mathrm{X}-100$, the slides were incubated with rabbit anti-BCL-2 and anti-Bax antibodies (1:500 diluted in PBS/ Tween; Cell Signaling Technology, Danvers, USA) for $1 \mathrm{~h}$ and with horseradish-conjugated donkey anti-rabbit IgG secondary antibodies (DAKO Corporation, Carpinteria, CA, USA) for another $1 \mathrm{~h}$ at $37^{\circ} \mathrm{C}$. The sections were eventually incubated in diaminobenzidine solution for peroxidase visualization.

\section{Statistical analysis}

One-way analysis of variance and two-tailed Student's $t$-test were employed to analyze the data, with $P$-value $<0.05$ considered statistically significant.

\section{Results \\ Preparation and characterization of TMZ-FaPEC@siRNA}

The folate-modified triblock copolymer PEG-PEI-PCL was synthesized through multistep reactions as outlined in Figure 1, and characterized by ${ }^{1} \mathrm{H}$ NMR spectrum (Figure 2). Apart from the signals attributed to PEG-PEI-PCL, the characteristic peaks for folate were clearly observed as well. The Fa-targeted and TMZ-loaded micelle (TMZ-FaPEC) was prepared by self-assembly of copolymer. The hydrophobic TMZ was encapsulated into the micelle core through hydrophobic interaction with the PCL matrix. According to dynamic light scattering (DLS) detection, TMZ-FaPEC possessed a diameter of $68 \pm 7 \mathrm{~nm}$ and a zeta potential of $+34.1 \pm 2.7 \mathrm{mV}$. A spherical morphology and relatively uniform size distribution were observed by transmission electron microscope (TEM; Figure $3 \mathrm{~A}$ and $\mathrm{B}$ ).

Owing to its high positive surface charge, TMZ-FaPEC easily complexed with BCL-2-siRNA to form the TMZ- and siRNA-coloaded micelle (TMZ-FaPEC@siRNA). Agarose gel electrophoresis revealed the siRNA complexation ability of Fa-PEC and nontargeted $\mathrm{PEC}$ at different $\mathrm{N} / \mathrm{P}$ ratios. It is well known that naked siRNA is negatively charged, which accounts for their motion in an electric field. Nevertheless, once siRNA has been complexed with positively charged polymers, such motion in an electric field will no longer be observed because of the loss of siRNA negative charge. Therefore, gel electrophoresis was performed to evaluate the siRNA complexation of cationic micelles. For both FaPEC and PEC, a complete retardation of siRNA motion was observed with N/P ratio above 4 (Figure 3C), indicating a thorough complexation of siRNA regardless of folate targeting.

The effect of siRNA complexation on the physical properties of TMZ-FaPEC@siRNA is shown in Figure 3A. According to DLS measurements, when N/P ratio increased, the zeta potential of micelle increased, whereas the particle size decreased, which indicated the successful siRNA complexation (Figure 3A). In other words, complexation of siRNA with the PEI chain of micelles led to more compact interlayer and thus decreased the micelle size, and meanwhile the complexed siRNA partially neutralized the positive charge of TMZ-FaPEC. At N/P 10, the micelle showed a relatively small size of $54 \pm 4 \mathrm{~nm}$ and a weak positive charge of $12.4 \pm 1.4 \mathrm{mV}$, which would facilitate the cell uptake of nanoparticles. Therefore, TMZ-FaPEC@siRNA of N/P 10 was selected for cell and animal studies. Under TEM observation, TMZ-FaPEC@siRNA of N/P 10 showed a spherical 


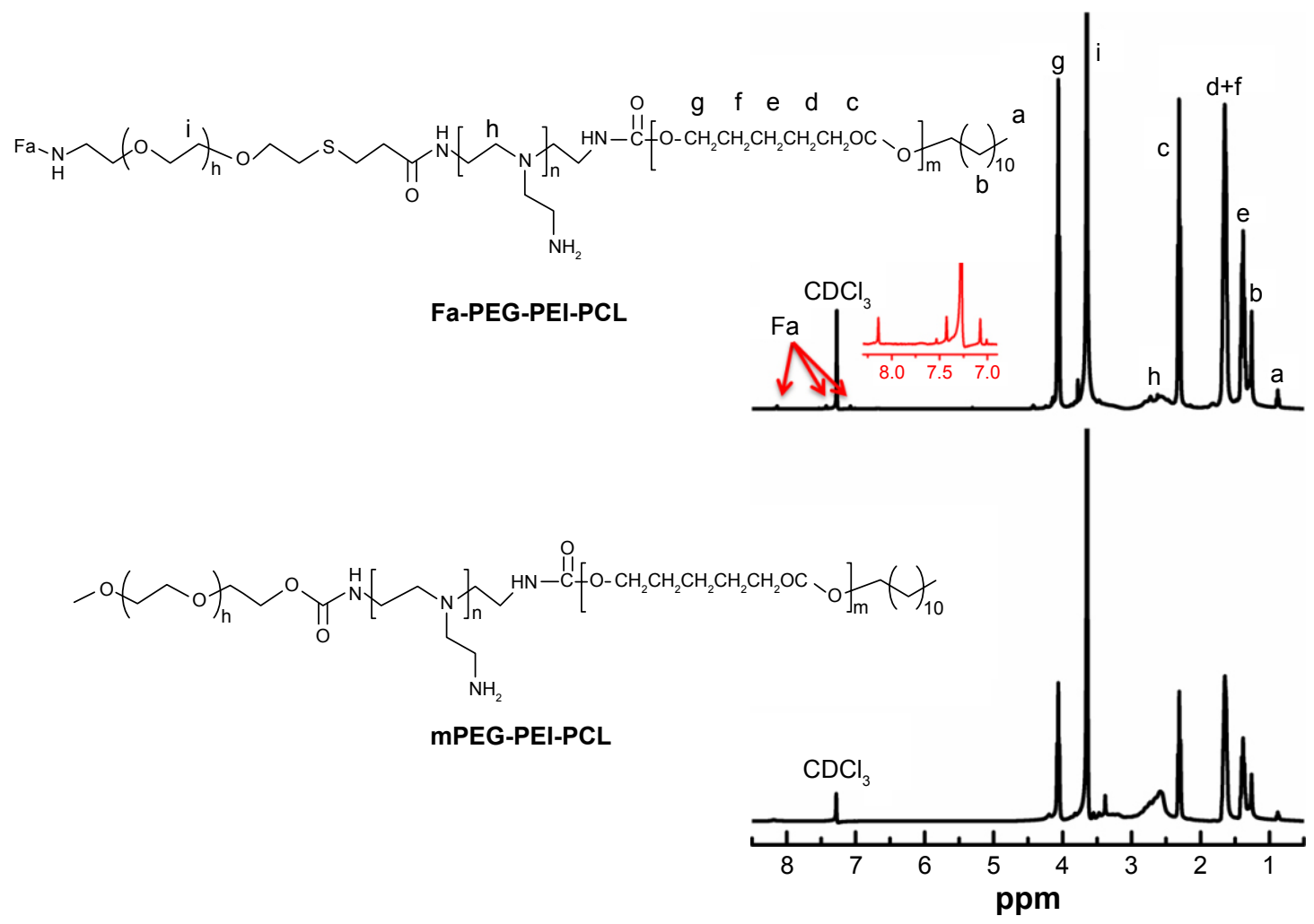

Figure 2 ' $\mathrm{H}$ NMR spectra of Fa-PEG-PEI-PCL and mPEG-PEI-PCL in $\mathrm{CDCl}_{3}$.

Note: The letters a-g indicate the chemical groups for peaks on the 'H NMR spectrum.

Abbreviations: Fa, folic acid; 'H NMR, protein nuclear magnetic resonance; PCL, poly(ع-caprolactone); PEG, poly(ethylene glycol), PEI, poly(ethylenimine).

nanostructure and average particle size around $56 \mathrm{~nm}$ consistent with the DLS determination. The TMZ loading content was 6.2\% for TMZ-FaPEC@siRNA of N/P 10.

The TMZ release from TMZ-FaPEC@siRNA of N/P 10 was investigated in vitro at $\mathrm{pH} 7.4$ and 5.0. As shown in Figure $3 \mathrm{D}, \mathrm{TMZ}$ was slowly released during a prolonged period. It is noteworthy that the TMZ release from TMZFaPEC@siRNA was slower than that from TMZ-FaPEC. Likely, the compact interlayer structure formed by PEI complexation with siRNA may hinder the drug diffusion out of micelle. Moreover, the TMZ release was obviously accelerated at $\mathrm{pH} 5.0$ for both the siRNA-complexed and siRNAfree micelles, as compared with the release at $\mathrm{pH}$ 7.4. One possible reason is that PEI turned more positively charged at $\mathrm{pH}$ 5.0, which may generate inter/intrachain repulsions to loosen the interlayer structure for easier TMZ diffusion.

\section{Study on intracellular distribution}

FR-mediated cell uptake of the micelle was investigated. To track the endocytosis of drug and siRNA, Coumarin 6 emitting green fluorescence and SCR-Cy3 (Cy3-labeled SCR) emitting red fluorescence were loaded into the micelle, and rat $\mathrm{C} 6$ cells as a typical glioma cell line were used for the study. ${ }^{23}$ The FR-enriched C6 cells incubated with Coumarin
6-FaPEC@SCR-Cy3 were observed with laser confocal microscopy and quantitative analysis was carried out with flow cytometry. As shown in Figure 4A, cells incubated with Coumarin 6-FaPEC@SCR-Cy3 for $2 \mathrm{~h}$ showed much stronger SCR-Cy3 (red) and Coumarin 6 (green) fluorescence than cells incubated with the nontargeting Coumarin 6-PEC@ SCR-Cy3. Consistently, the quantitative flow cytometry analysis revealed that both the $\mathrm{Cy} 3$-positive and Coumarin 6-positive populations increased significantly when the cells were incubated with Coumarin 6-FaPEC@SCR-Cy3 rather than Coumarin 6-PEC@SCR-Cy3 (Figure 4B). Moreover, although the fluorescence intensities of Coumarin 6 and SCR-Cy3 were different, distributions of Coumarin 6 fluorescence and SCR-Cy3 fluorescence were nearly identical, that is, mostly around the nuclei and within the cytoplasm. Orange stains in the merged image were generated as a result of red and green fluorescence overlapping (Figure 4A). In the presence of $1 \mathrm{mg} / \mathrm{L}$ folate, the cell uptake level of Coumarin 6-FaPEC@SCR-Cy3 was obviously decreased according to laser confocal microscopic analysis. Quantitatively, the ratio of Cy3-positive and Coumarin 6-positive cells was almost decreased to the same level as that in cells treated with Coumarin 6-PEC@SCR-Cy3, according to flow cytometric analysis. These results strongly suggested that FR mediated 
A
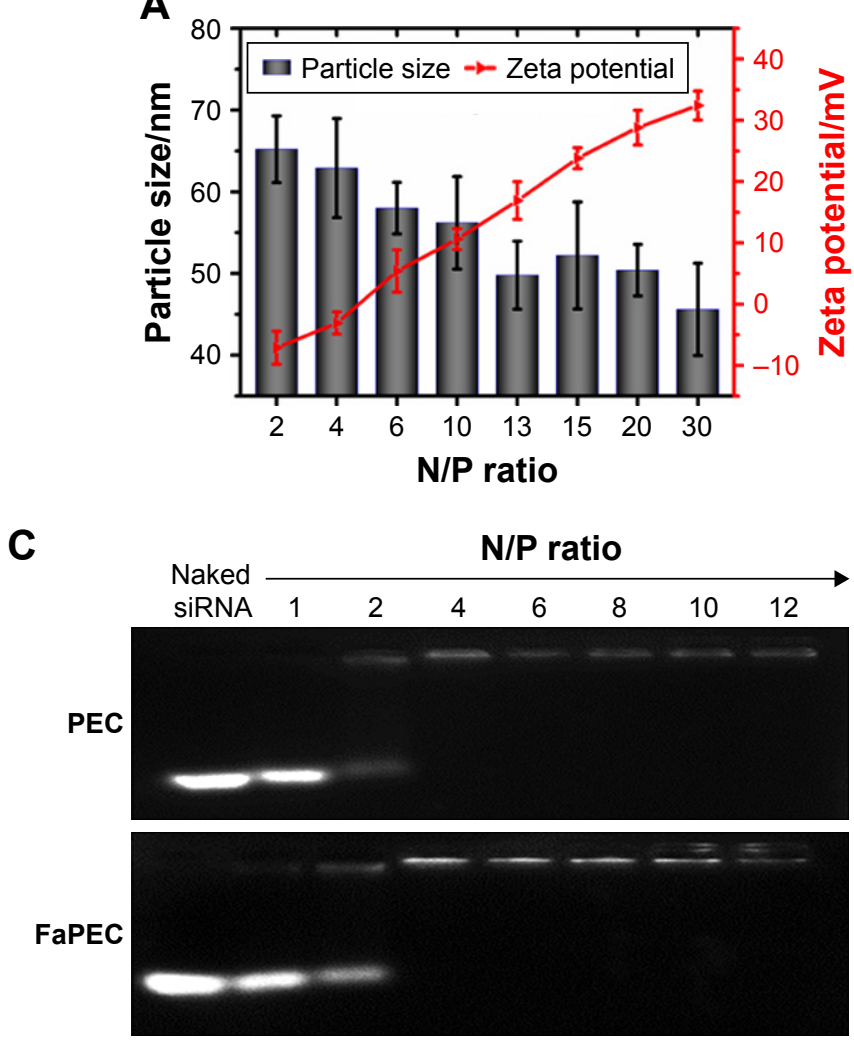

B
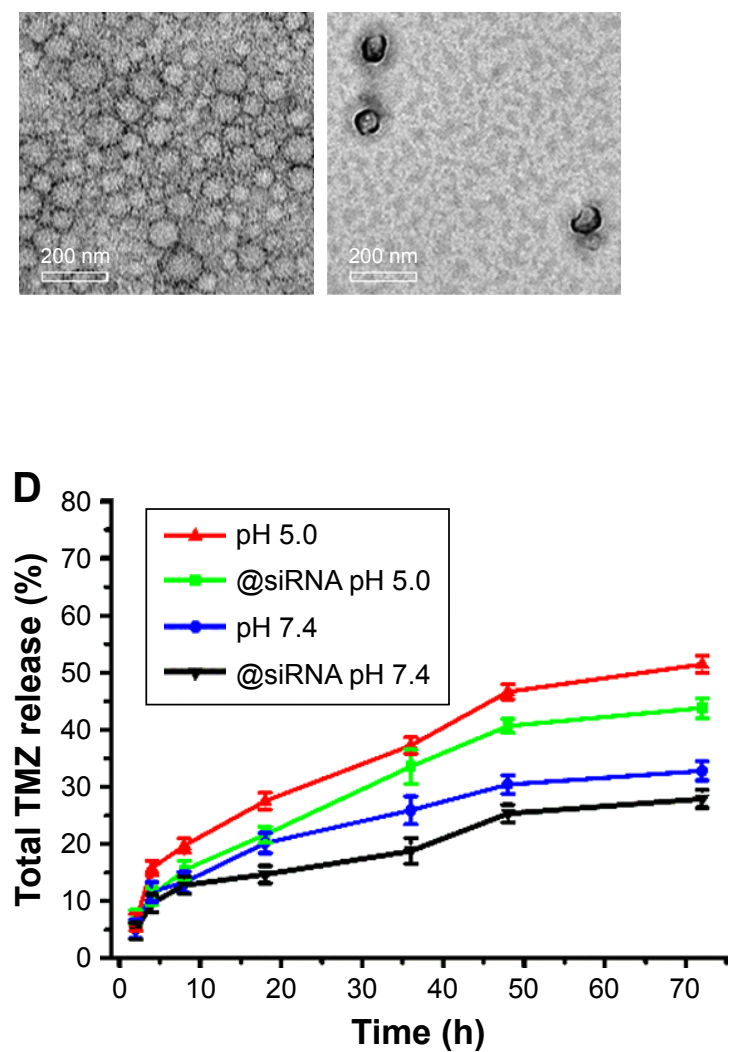

Figure 3 (A) Change of particle size and zeta potential of TMZ-FaPEC@siRNA micelle against N/P ratio. (B) TEM images of TMZ-FaPEC micelle (left) and TMZ-FaPEC@ siRNA micelle $(\mathrm{N} / \mathrm{P}=10)$ (right) at $\mathrm{pH} 7.4$. All samples were stained with $0.5 \%$ uranyl acetate for I min. Scale bar: $200 \mathrm{~nm}$; magnification $\times 50,000$. (C) Electrophoretic mobility of SCR in agarose gel after complexation with FaPEC or PEC at N/P ratio ranging from I to I2. (D) In vitro TMZ release from TMZ-FaPEC@SCR and TMZ-FaPEC@siRNA micelles at $\mathrm{pH} 7.4$ and $\mathrm{pH} 5.0$.

Abbreviations: N/P, nitrogen in branched PEI to phosphorus in siRNA; SCR, scramble siRNA; TEM, transmission electron microscope; TMZ, temozolomide.

an effective endocytosis of Coumarin 6-FaPEC@SCR-Cy3, resulting in an effective codelivery of siRNA and drugmimicking Coumarin 6 into the $\mathrm{C} 6$ cells.

\section{In vitro anticancer activity of TMZ-FaPEC@siRNA}

The cytotoxicities of FaPEC and PEC carrying SCR were measured by CCK8 assay. As shown in Figure 5A, C6 cells incubated with FaPEC@SCR and PEC@SCR displayed viabilities above $90 \%$ even at a high polymer concentration of $100 \mu \mathrm{g} / \mathrm{mL}$, indicating low cytotoxicity of carrier, which was important for in vivo application. For cells incubated with TMZ-loaded micelles, a negative correlation was observed between the cell viability and TMZ concentration. Moreover, TMZ-FaPEC@siRNA showed obviously higher cytotoxicity than both TMZ-FaPEC and TMZ-PEC (Figure 5B). For example, at the TMZ concentration of $120 \mu \mathrm{g}$, cells treated with TMZ-FaPEC@siRNA showed $49.09 \%$ viability, whereas cells treated with other micelles exhibited $>60 \%$ viability.
Flow cytometry was conducted to quantitatively evaluate cell apoptosis after treatment with nanocomplexes. As shown in Figure 6, the control cells without treatment showed an extremely low apoptosis rate of $2.8 \%$. In contrast, TMZFaPEC@siRNA treatment induced the highest cell apoptosis rate around $66 \%$ among all treatment groups. The TMZPEC@siRNA, TMZ-FaPEC@SCR, FaPEC@siRNA, and free TMZ treatments only resulted in cell apoptosis rates of $51.2 \%, 44.9 \%, 34.3 \%$, and $15.8 \%$, respectively. These results further demonstrated that the anticancer effect in vitro was elevated by combination therapy because the codelivered TMZ and BCL-2 siRNA jointly induced cancer cell apoptosis.

\section{In vitro gene silencing effect}

An upregulated expression of BCL-2 was discovered in glioma, which may account for the enhanced proliferation and suppressed apoptosis of glioma cells. ${ }^{24}$ Thus, BCL-2 gene silencing in C6 cells was attempted through RNA interference. The expression levels of proapoptotic Bax gene in the 

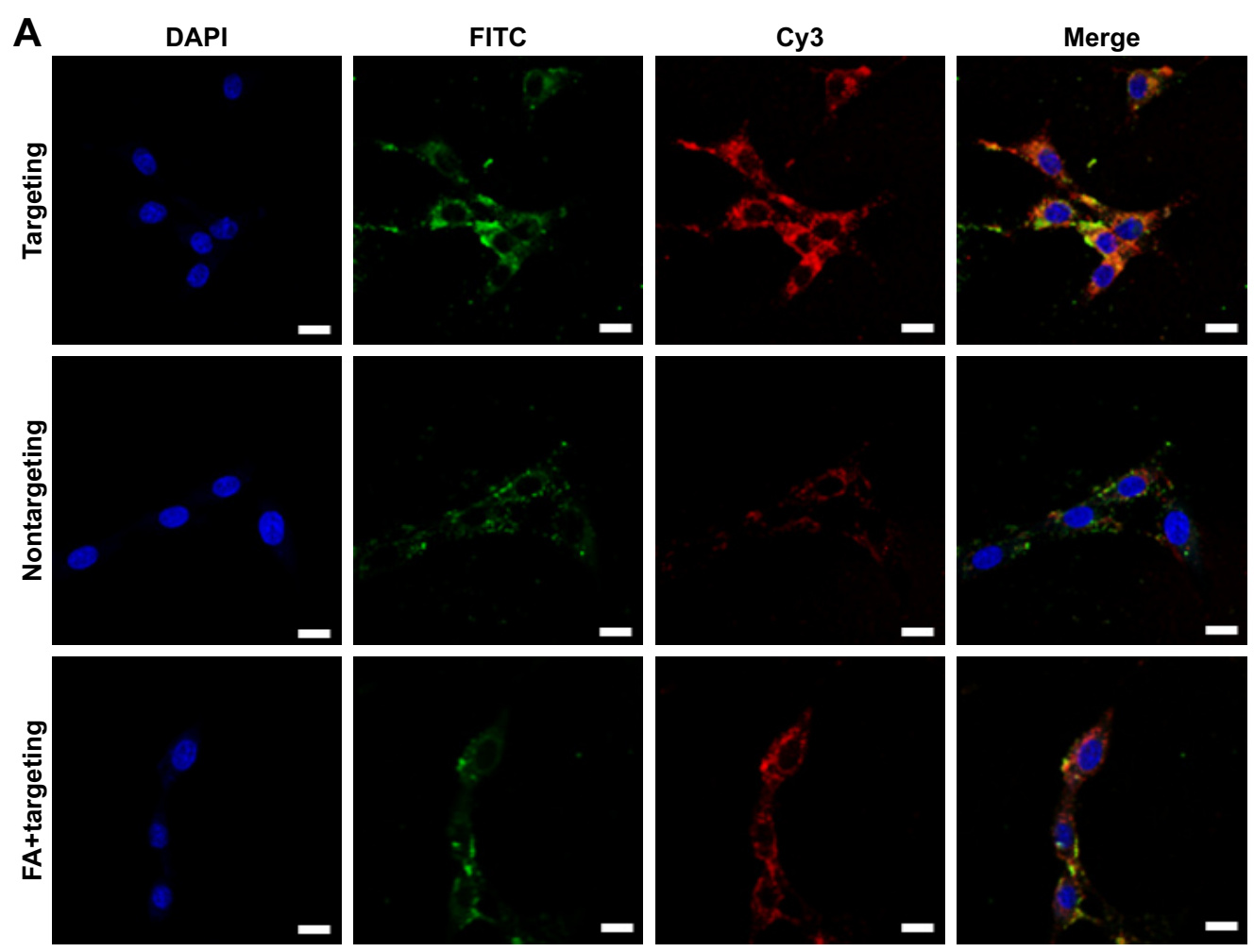

B

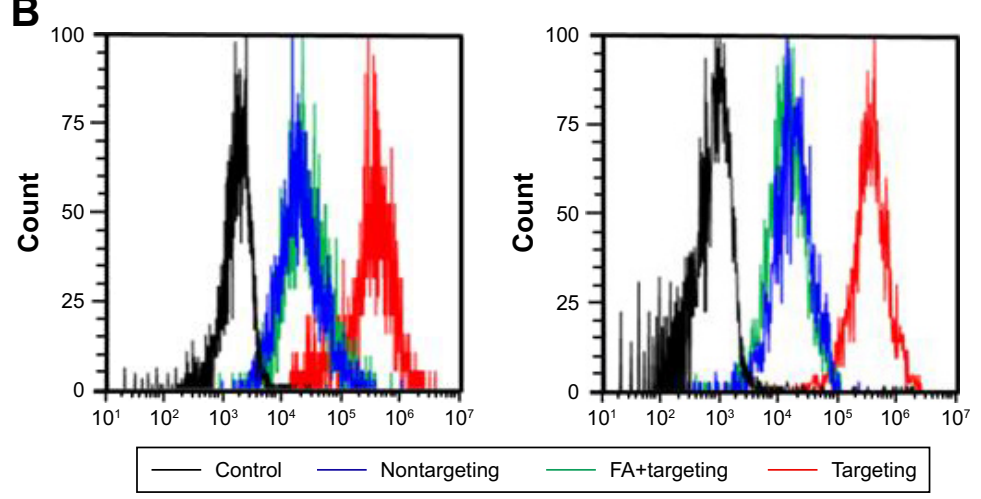

Figure 4 (A) CLSM images of C6 cells incubated with Coumarin 6-FaPEC@Cy3-SCR (targeting), Coumarin 6-PEC@Cy3-SCR (nontargeting), and Coumarin 6-FaPEC@ Cy3-SCR plus I mg/L Fa (Fa+targeting). Nuclei are blue because of DAPI staining, red fluorescence indicates Cy3 (siRNA), green fluorescence indicates Coumarin 6 (TMZ), and the yellow stains indicate overlapping of red and green fluorescence. (B) Quantitative determination of Cy3 and FITC (Coumarin 6) positive cells by flow cytometry. Coumarin 6 loading content: $8 \%$. Scale bars: $10 \mu \mathrm{m}$; magnification $\times 630$. Incubation time: $2 \mathrm{~h}$. Dose: $20 \mathrm{nM}$ siRNA. The N/P ratio of all micelles was I0.

Abbreviations: CLSM, confocal laser scanning microscopy; DAPI, 4',6-diamidino-2-phenylindole; FITC, fluorescein isothiocyanate; N/P, nitrogen in branched PEI to phosphorus in siRNA; SCR, scramble siRNA; TMZ, temozolomide.

cells were also measured to comprehensively analyze the C6 cell apoptosis induced by TMZ-FaPEC@siRNA. The transcription and translation of both BCL-2 and Bax were evaluated using RT-PCR and Western blotting, respectively. As demonstrated by RT-PCR, the treatment with TMZFaPEC@siRNA significantly decreased the expression of BCL-2 at mRNA level, as compared with TMZ-PEC@ siRNA, TMZ-FaPEC@SCR, FaPEC@siRNA, and free TMZ (Figure 7A). Meanwhile, cells incubated with TMZFaPEC@siRNA showed much higher level of Bax mRNA $(243.9 \% \pm 3.6 \%)$ than cells treated with TMZ-FaPEC@SCR (130.5\% $\pm 2.4 \%)$, FaPEC@siRNA (118.9\% $\pm 1.6 \%)$, and TMZ
$(110.7 \% \pm 3.3 \%$ ) (Figure 7B). The results of Western blotting assays were consistent with the RT-PCR data, as shown in Figure $7 \mathrm{C}$ and D. These results clearly showed that BCL-2 siRNA and TMZ codelivered by the folate-modified micelle acted together on $\mathrm{C} 6$ cells to induce apoptosis by silencing BCL-2 and meanwhile upregulating the expression of Bax.

\section{Therapeutic effect in vivo}

In vivo experiments were carried out to evaluate the therapeutic effect of FaPEC carrying both TMZ and BCL-2 siRNA. During the experimental process, the rats in the control group drastically lost weight, with their hair appearing 
A

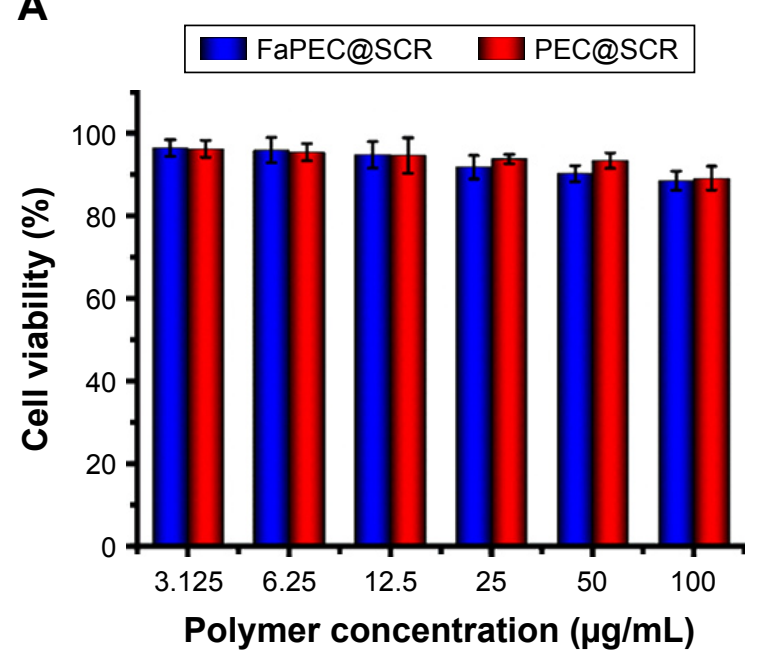

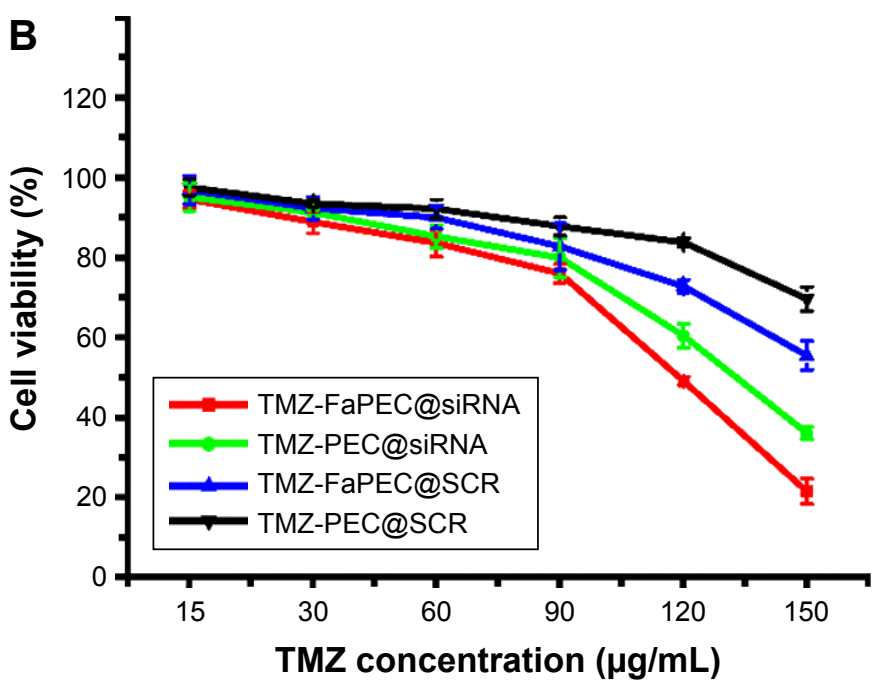

Figure 5 (A) In vitro cytotoxicities of TMZ-free PEC@SCR and FaPEC@SCR at various concentrations in C6 cells. Incubation time: 48 h. BCL-2 siRNA concentration: 20 nM. (B) In vitro cytotoxicities of TMZ-FaPEC@siRNA, TMZ-PEC@siRNA, TMZ-FaPEC@SCR, and TMZ-PEC@SCR at various concentrations in C6 cells. Incubation time: 48 h. BCL-2 siRNA concentration: $20 \mathrm{nM}$.

Abbreviations: TEM, transmission electron microscope; SCR, scramble siRNA; TMZ, temozolomide.

lackluster and activity level obviously decreased. In contrast, most of these symptoms were alleviated in the group of TMZFaPEC@siRNA. The tumor volume at 25 days after the first treatment was determined by MRI, which clearly showed the different effects of various treatments on the glioma growth in rat brain (Figure $8 \mathrm{~A}$ ). The tumor in rats receiving PBS grew to $262.93 \pm 19.46 \mathrm{~mm}^{3}$. In contrast, the tumor volumes in rats receiving TMZ-FaPEC@SCR,FaPEC@siRNA, and
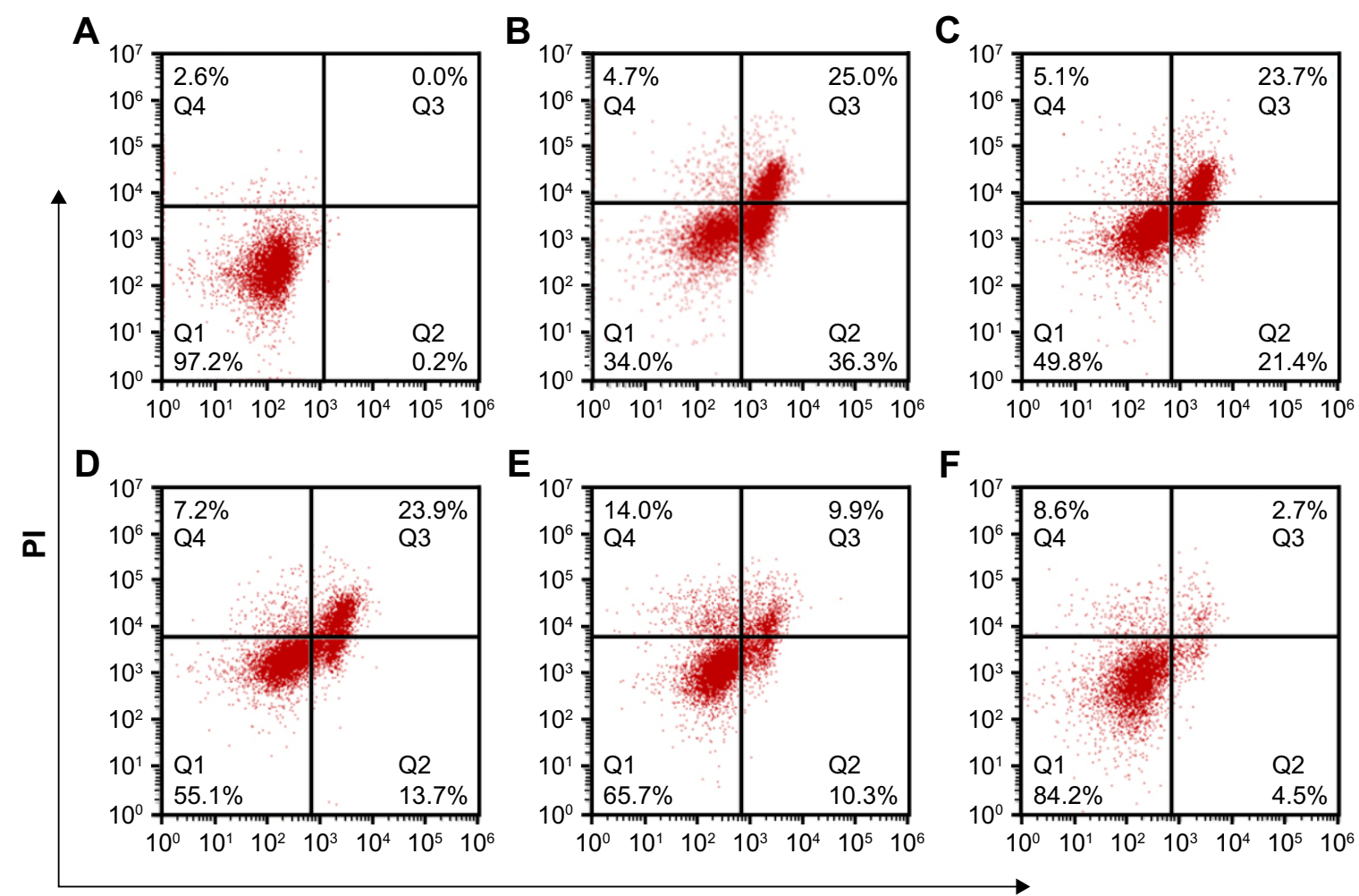

\section{Annexin V-FITC}

Figure 6 Quantitative analysis of apoptotic C6 cells using Annexin V-FITC and PI flow cytometry assay at $48 \mathrm{~h}$ after different treatments.

Notes: BCL-2 siRNA concentration: $20 \mathrm{nM}$. TMZ dose: $120 \mu \mathrm{g}$. Q1: normal viable cells; Q2: early-stage apoptotic cells; Q3: late-stage apoptotic cells; Q4: necrotic cells. (A) Control, (B) TMZ-FaPEC@siRNA, (C) TMZ-PEC@siRNA, (D) TMZ-FaPEC@SCR, (E) FaPEC@siRNA, and (F) free TMZ.

Abbreviations: FITC, fluorescein isothiocyanate; PI, propidium iodide; SCR, scramble siRNA; TMZ, temozolomide. 

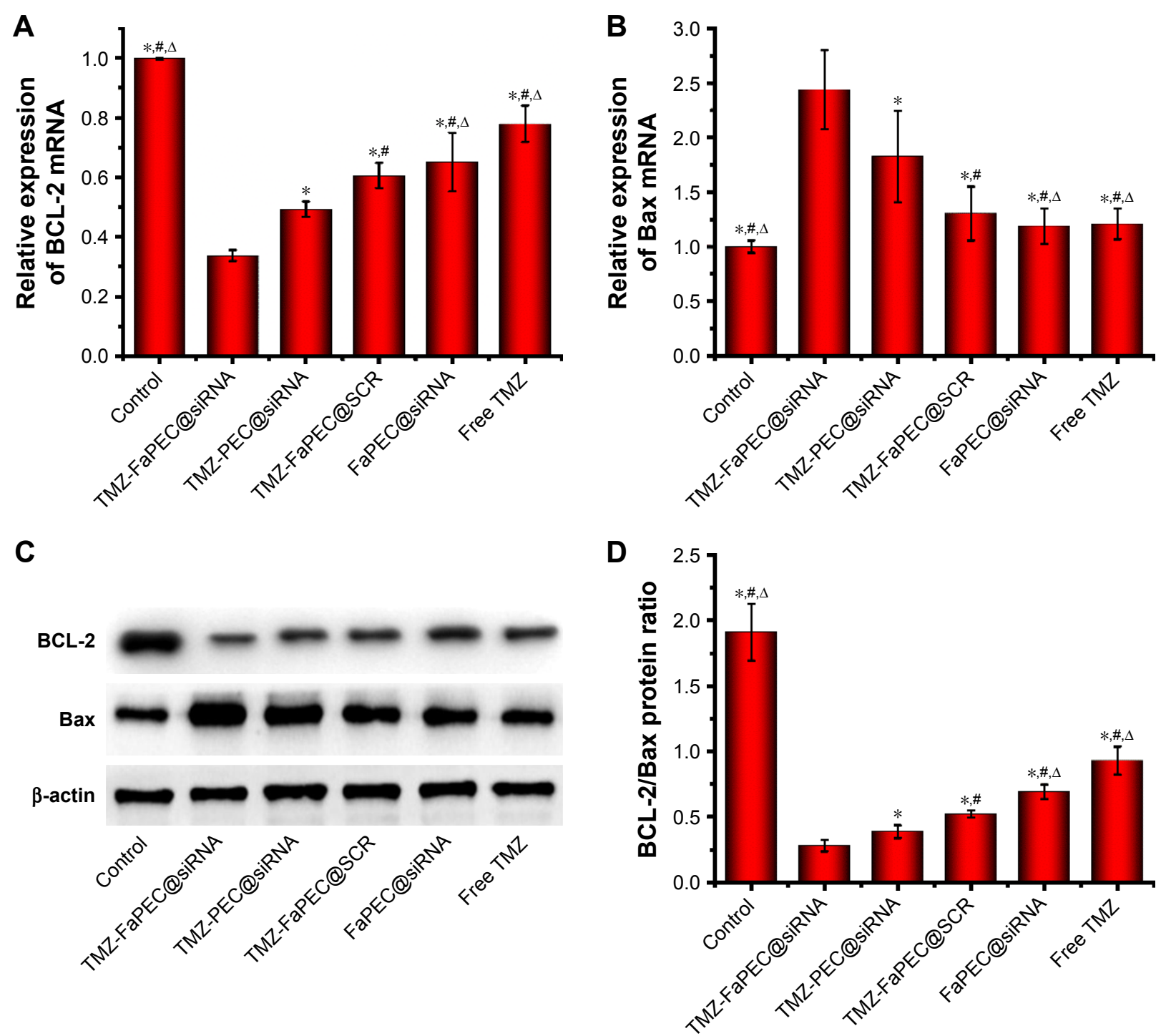

Figure 7 (A) Expressions of BCL-2 at mRNA level determined by RT-PCR in C6 cells receiving different treatments. *P $<0.05$ vs TMZ-FaPEC@siRNA; \#P $<0.05$ vs TMZ-PEC@siRNA; ${ }^{\triangle P}<0.05$ vs TMZ-FaPEC@SCR. (B) Expressions of Bax at mRNA levels determined by RT-PCR in C6 cells receiving different treatments. *P < 0.05 vs TMZ-FaPEC@siRNA; ${ }^{P} P<0.05$ vs TMZ-PEC@siRNA; ${ }^{\triangle P}<0.05$ vs TMZ-FaPEC@SCR. (C) Western blotting analysis for protein expression levels of BCL-2 and Bax in C6 cells receiving different treatments. (D) Statistical analysis of $B C L-2 / B a x$ protein ratios in image $C$ showing Western blotting. ${ }^{* P}<0.05$ vs TMZ-FaPEC @siRNA; ${ }^{P} P<0.05$ vs TMZ-PEC@siRNA; ${ }^{\Delta P}<0.05$ vs TMZ-FaPEC@SCR.

Abbreviations: SCR, scramble siRNA; TMZ, temozolomide.

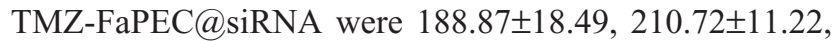
and $82.25 \pm 11.28 \mathrm{~mm}^{3}$, respectively (Figure $8 \mathrm{~B}$ ). According to these results, although the siRNA or TMZ alone treatment mediated by the folate-targeted nanocarrier was able to inhibit glioma growth to some extent, the combinative therapy was apparently much more effective. In addition, the rats receiving TMZ-PEC@siRNA showed a tumor volume of $139.04 \pm 20.60 \mathrm{~mm}^{3}$, indicating a much lower therapeutic effect of the nontargeted combinative therapy than the folate-targeted one. These results demonstrated that the combinative therapy of siRNA and TMZ would be beneficial in the treatment of glioma and the therapeutic effect could be further improved with the folate-targeted codelivery nanocarrier.
As shown in Figure 8C, the survival rates of rats varied with therapeutic regimen. The control group exhibited the lowest survival rate, with no rats surviving after 28 days. In contrast, the highest survival rate appeared in the group treated with TMZ-FaPEC@siRNA, and the other three treatments also exhibited conspicuous therapeutic effects. No animal receiving FaPEC@siRNA or TMZ-FaPEC@SCR survived longer than 35 days and all animals receiving TMZPEC@siRNA died within 39 days. Excitingly, the survival rate of rats receiving TMZ-FaPEC@siRNA group was still as high as $50 \%$ at day 39 .

$H \& E$ stain visualized the histologic changes of the glioma (Figure 9). The sections from the control group showed obvious hypercellularity hemorrhage foci as well as polymorphic 
A
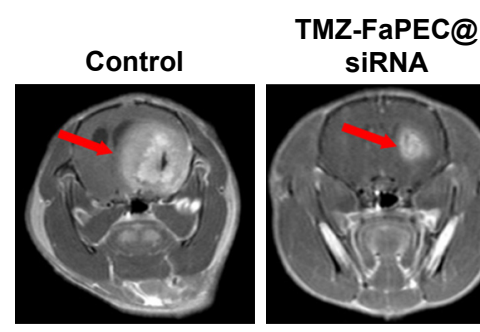
SiRNA
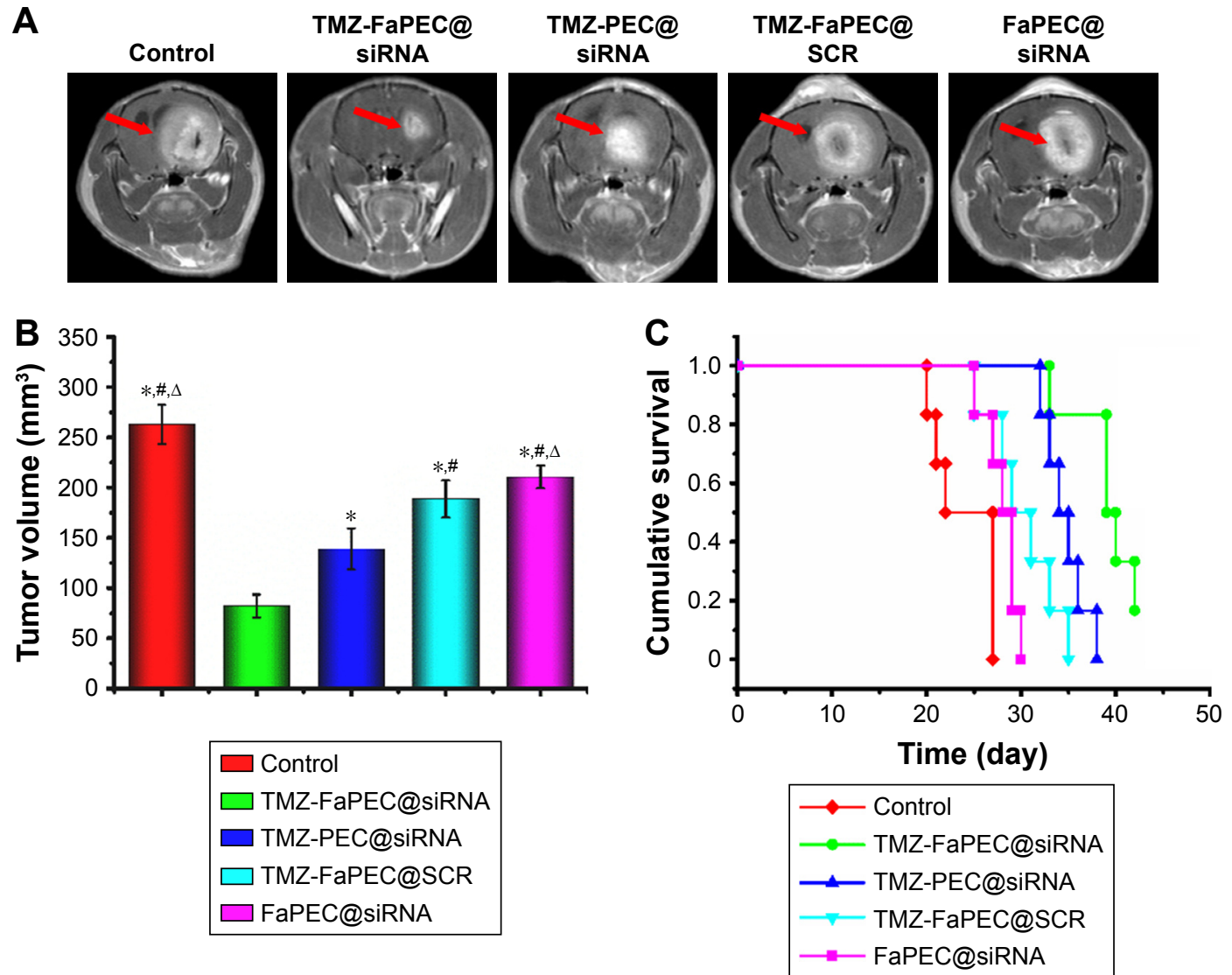

Figure 8 (A) Visualization of tumor growth inhibition (tumor indicated with red arrow) in rat bearing C6 glioma after injections of various formulations. (B) Determination of mean tumor volume, using magnetic resonance imaging at the time point 25 days after the first injection. *P $<0.05$ vs TMZ-FaPEC@siRNA; ${ }^{2}<0.05$ vs TMZ-PEC@siRNA; $\triangle P<0.05$ vs TMZ-FaPEC@SCR. (C) Cumulative survival of rats bearing C6 glioma in brain after receiving injection of different formulations ( $\mathrm{n}=8$ ). Doses per injection: $120 \mu \mathrm{g} \mathrm{TMZ} / \mathrm{kg}$ body weight and I.6 $\mu \mathrm{g}$ siRNA/kg body weight. Abbreviations: SCR, scramble siRNA; TMZ, temozolomide.

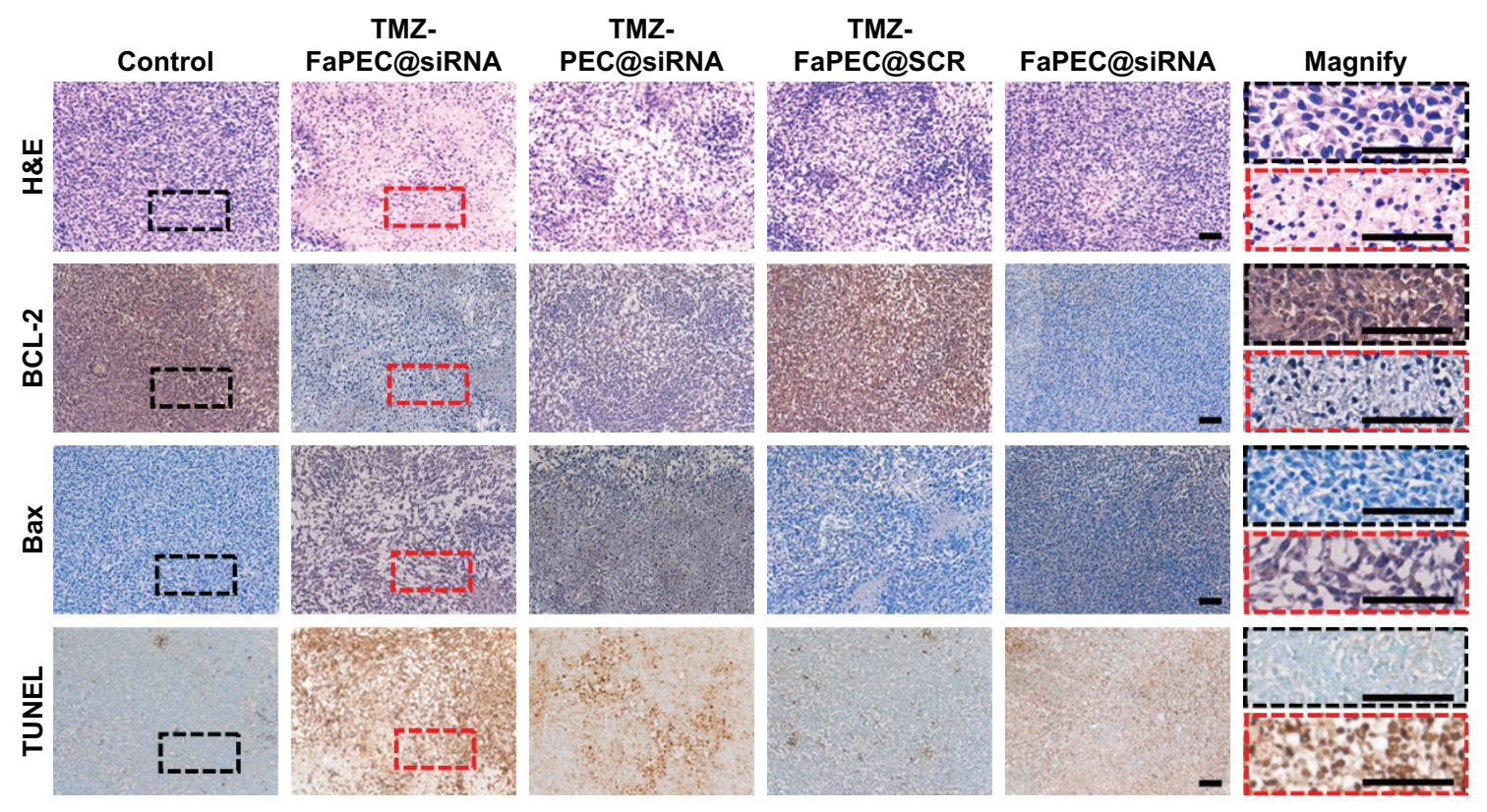

Figure 9 Ex vivo immunohistochemical and TUNEL analyses of C6 glioma sections from rats receiving different formulations (25 days after the first treatment).

Notes: In the immunohistochemical assay, the brown and blue stains indicated BCL-2 or Bax protein and nuclei, respectively. In TUNEL analysis, brown and green stains indicated apoptotic and normal cells, respectively. Magnified images of the black and red rectangular areas in control and TMZ-FaPEC@siRNA groups are shown on the right side. Scale bars: $50 \mu \mathrm{m}$; magnification $\times 200$.

Abbreviations: H\&E, hematoxylin and eosin; TMZ, temozolomide; TUNEL, terminal deoxynucleotidyl transferase dUTP nick end labeling. 
nuclei. In contrast, tumor cell necrosis was found to be the most remarkable in the group receiving TMZ-FaPEC@, siRNA, revealing the optimized therapeutic effect achieved by the combinative therapy through folate-targeted codelivery. Finally, immunohistochemical staining was carried out to evaluate the effects of different treatments on the expressions of BCL-2 and Bax proteins in tumor tissues. As can be seen in Figure 9, BCL-2 or Bax protein was stained brown while the blue stains indicated cell nuclei. In comparison with the control group, after the rats were treated with TMZ-FaPEC@ SCR and FaPEC@siRNA, the expression of BCL-2 was downregulated, whereas the expression of Bax was upregulated. Moreover, both the lowest level of BCL-2 protein and the highest level of Bax protein were observed in the group receiving TMZ-FaPEC@siRNA, which suggested that C6 cells turned highly apoptotic upon the combinative therapy of TMZ and siRNA codelivered by Fa-PEC. This notion was verified by directly measuring cell apoptosis using TUNEL assay (Figure 9). Compared with BCL-2 siRNA or TMZ treatment alone, the combinative therapy exhibited a higher potency to induce the apoptosis of glioma cells, based on which an improved therapeutic outcome was obtained by folate-targeted codelivery of siRNA and TMZ.

\section{Discussion}

TMZ is known as the first-line drug in glioma chemotherapy, which induces cancer cell apoptosis by intercalating into DNA and then inhibiting macromolecular biosynthesis. ${ }^{25}$ The efficacy of TMZ-based single-drug therapy is limited because of drug resistance. ${ }^{10,26}$ RNAi using single- or doublestranded RNA has found great application potentials in the treatments of various diseases because of its sequence specificity and enhanced safety as it only interferes with the posttranscriptional process. ${ }^{27,28}$ The combination of TMZ chemotherapy and gene therapy is regarded as a promising strategy for glioma treatment. ${ }^{25}$ The major challenge is to develop a vector with good biocompatibility and desirable delivery efficiency. ${ }^{29}$

Herein, a triblock copolymer FaPEC was used to encapsulate TMZ and siRNA through intermolecular hydrophobic interaction and electrostatic complexation, respectively. Considering the overexpressed FR on C6 cells, FaPEC was modified with folate in order to facilitate the cellular uptake of the codelivery system. The results showed that this dual functional nanoplatform provided remarkable efficacy in codelivering TMZ and siRNA into C6 cells, and meanwhile the carrier itself exhibited low toxicity. A much better delivery efficiency for TMZ and siRNA was achieved by folate-modified nanocomplexes compared with the nontargeting ones, which indicated the folate-guided codelivery event. ${ }^{19,22,30}$ CCK8 and apoptosis assays showed that the combinative treatment of TMZ and siRNA had a potent effect on cell apoptosis by jointly suppressing the expression of BCL-2 and meanwhile upregulating the expression of Bax, which resulted in the elevated anticancer effect in vitro. As BCL-2 gene is frequently overexpressed in glioma cells, the folate-targeted nanomedicine appeared as a proper candidate to further explore the combined therapeutic effect in vivo. ${ }^{12,26}$

In vivo study showed that codelivery of BCL-2 siRNA and TMZ significantly inhibited the growth of glioma. The survival rate of glioma-bearing rats was much elevated when BCL-2 siRNA and TMZ were jointly administered than used alone, and with folate-targeted strategy, the therapeutic effect would be further enhanced. Immunohistochemical assay clearly showed that BCL-2 siRNA and TMZ codelivered by the folate-targeted micelle acted together to induce C6 cell apoptosis, which could account for the achieved significant tumor inhibition and survival benefit.

We chose direct injection rather than systemic delivery for better delivery efficiency. Due to the existence of BBB, systemic delivery as a means to deliver the folate-targeted nanocarriers to brain tissue was extremely difficult although FR is a suitable glioma target in vivo. ${ }^{31}$ However, the unfavorable effect of BBB on delivery may be bypassed through direct local injection. Moreover, nanomedicine applied through intracranial injection may have good chance to be sequestrated inside brain because of the effects of both folatetargeting and BBB-blocked washout. ${ }^{32}$ Therefore, the therapeutic effect would probably be impaired if the direct delivery method is changed to systemic delivery. ${ }^{33}$ Nevertheless, a careful selection of targeting molecules with high specificity is worthwhile in further systemic delivery studies.

\section{Conclusion}

A polymeric micelle based on the triblock copolymer Fa-PEG-PEI-PCL was prepared, with TMZ encapsulated in the core and BCL-2 siRNA complexed by the cationic layer. Both in vitro and in vivo studies demonstrated remarkably efficient codelivery of the two therapeutic agents into C6 glioma cells. Consequently, antiapoptotic BCL-2 gene was effectively silenced, and meanwhile the proapoptotic Bax gene was effectively activated, which led to the most significant tumor apoptosis. In animal study, after the combinative therapy of TMZ and BCL-2 siRNA mediated by folate-targeted nanocarrier, the tumor growth was obviously 
inhibited and the survival rate of rats bearing orthotropic C6 glioma was dramatically enhanced. Our study shows that the combinative BCL-2 siRNA and TMZ treatment using the folate-targeted micelle might have promising prospects in glioma treatment.

\section{Acknowledgment}

This work was supported by the National Natural Science Foundation of China (U1401242), National Basic Research Program of China (2015CB755500), Natural Science Foundation of the Guangdong Province (2014A030312018), and the Guangdong Innovative and Entrepreneurial Research Team Program (2013S086).

\section{Disclosure}

The authors report no conflicts of interest in this work.

\section{References}

1. Wang W, Shi G, Ma B, Hao X, Dong X, Zhang B. Chemotherapy for adults with malignant glioma: a systematic review and network metaanalysis. Turk Neurosurg. 2017;27:174-181.

2. Ziu M, Kalkanis SN, Gilbert M, Ryken TC, Olson JJ. The role of initial chemotherapy for the treatment of adults with diffuse low grade glioma: a systematic review and evidence-based clinical practice guideline. J Neurooncol. 2015;125:585-607.

3. Juratli TA, Schackert G, Krex D. Current status of local therapy in malignant gliomas - a clinical review of three selected approaches. Pharmacol Ther. 2013;139:341-358.

4. An den Bent MJ, Baumert B, Erridge SC, et al. Interim results from the CATNON trial (EORTC study 26053-22054) of treatment with concurrent and adjuvant temozolomide for $1 \mathrm{p} / 19 \mathrm{q}$ non-co-deleted anaplastic glioma: a phase 3, randomised, open-label intergroup study. Lancet 2017;390:1645-1653.

5. Pinheiro R, Braga C, Santos G, et al. Targeting gliomas: can a new alkylating hybrid compound make a difference? ACS Chem Neurosci. 2017;8:50-59.

6. Feng X, Zhang B, Wang J, Xu X, Lin N, Liu H. Adenovirus-mediated transfer of siRNA against basic fibroblast growth factor mRNA enhances the sensitivity of glioblastoma cells to chemotherapy. Med Oncol. 2011;28:24-30.

7. Whitehead KA, Langer R, Anderson DG. Knocking down barriers: advances in sirna delivery. Nat Rev Drug Discov. 2009;8:129-138.

8. Juliano R, Alam MR, Dixit V, Kang H. Mechanisms and strategies for effective delivery of antisense and siRNA oligonucleotides. Nucleic Acids Res. 2008;36:4158-4171.

9. Liu X, Madhankumar AB, Slagle-Webb B, Sheehan JM, Surguladze N, Connor JR. Heavy chain ferritin sirna delivered by cationic liposomes increases sensitivity of cancer cells to chemotherapeutic agents. Cancer Res. 2011;71:2240-2249.

10. Hombach-Klonisch S, Mehrpour M, Shojaei S, et al. Glioblastoma and chemoresistance to alkylating agents: involvement of apoptosis, autophagy, and unfolded protein response. Pharmacol Ther. 2018;184: 13-41.

11. Straten P, Andersen MH. The anti-apoptotic members of the bcl-2 family are attractive tumor-associated antigens. Oncotarget. 2010;1: 239-245.
12. Stegh AH, Brennan C, Mahoney JA, et al. Glioma oncoprotein bcl2112 inhibits the p53 tumor suppressor. Genes Dev. 2010;24:2194-2204.

13. Jensen SA, Day ES, Ko CH, et al. Spherical nucleic acid nanoparticle conjugates as an RNAi-based therapy for glioblastoma. Sci Transl Med. 2013;5:209ra152.

14. Yuan Y, Gong F, Cao Y, Chen W, Cheng D, Shuai X. Biodegradable multiamine polymeric vector for sirna delivery. J Biomed Nanotechnol. 2015;11:668-679.

15. Yin H, Kanasty RL, Eltoukhy AA, Vegas AJ, Dorkin JR, Anderson DG. Non-viral vectors for gene-based therapy. Nat Rev Genet. 2014;15: 541-555.

16. Islam MA, Park TE, Singh B, et al. Major degradable polycations as carriers for DNA and siRNA. J Control Release. 2014;193:74-89.

17. Kim JH, Kim YK, Arash MT, et al. Galactosylation of chitosan-graftspermine as a gene carrier for hepatocyte targeting in vitro and in vivo. J Nanosci Nanotechnol. 2012;12:5178-5184.

18. Hong CA, Nam YS. Functional nanostructures for effective delivery of small interfering rna therapeutics. Theranostics. 2014;4:1211-1232.

19. Zou S, Cao N, Cheng D, et al. Enhanced apoptosis of ovarian cancer cells via nanocarrier-mediated codelivery of sirna and doxorubicin. Int J Nanomedicine. 2012;7:3823-3835.

20. Weitman SD, Frazier KM, Kamen BA. The folate receptor in central nervous system malignancies of childhood. J Neurooncol. 1994;21: 107-112.

21. Brockmann MA, Papadimitriou A, Brandt M, et al. Inhibition of intracerebral glioblastoma growth by local treatment with the scatter factor/ hepatocyte growth factor-antagonist nk4. Clin Cancer Res. 2003;9: 4578-4585.

22. Cheng D, Cao N, Chen J, Yu X, Shuai X. Multifunctional nanocarrier mediated co-delivery of doxorubicin and sirna for synergistic enhancement of glioma apoptosis in rat. Biomaterials. 2012;33:1170-1179.

23. Barth RF, Kaur B. Rat brain tumor models in experimental neuro-oncology: the c6, 91, t9, rg2, f98, bt4c, rt-2 and cns-1 gliomas. JNeurooncol. 2009; 94:299-312.

24. Latha K, Li M, Chumbalkar V, et al. Nuclear egfrviii-stat5b complex contributes to glioblastoma cell survival by direct activation of the bcl-xl promoter. Int J Cancer. 2013;132:509-520.

25. Zhitao J, Long L, Jia L, Yunchao B, Anhua W. Temozolomide sensitizes stem-like cells of glioma spheres to trail-induced apoptosis via upregulation of casitas b-lineage lymphoma (c-cbl) protein. Tumour Biol. 2015;36:9621-9630.

26. Wakabayashi T. Clinical trial updates for malignant brain tumors. Rinsho Shinkeigaku. 2011;51:853-856.

27. Liu X, Liu C, Zhou J, et al. Promoting sirna delivery via enhanced cellular uptake using an arginine-decorated amphiphilic dendrimer. Nanoscale. 2015;7:3867-3875.

28. Gilleron J, Paramasivam P, Zeigerer A, et al. Identification of siRNA delivery enhancers by a chemical library screen. Nucleic Acids Res. 2015; 43:7984-8001.

29. Markman JL, Rekechenetskiy A, Holler E, Ljubimova JY. Nanomedicine therapeutic approaches to overcome cancer drug resistance. Adv Drug Deliv Rev. 2013;65:1866-1879.

30. Butowski N, Colman H, De Groot JF, et al. Orally administered colony stimulating factor 1 receptor inhibitor PLX3397 in recurrent glioblastoma: an Ivy foundation early phase clinical trials consortium phase II study. Neuro Oncol. 2016;18:557-564.

31. Oberoi RK, Parrish KE, Sio TT, Mittapalli RK, Elmquist WF, Sarkaria JN. Strategies to improve delivery of anticancer drugs across the blood-brain barrier to treat glioblastoma. Neuro Oncol. 2016;18:27-36.

32. Wang S, Meng Y, Li C, et al. Receptor-mediated drug delivery systems targeting to glioma. Nanomaterials (Basel). 2015;6.

33. Wilhelm S, Tavares AJ, Dai Q, et al. Analysis of nanoparticle delivery to tumours. Nature. 2016;1-29. 


\section{Publish your work in this journal}

The International Journal of Nanomedicine is an international, peerreviewed journal focusing on the application of nanotechnology in diagnostics, therapeutics, and drug delivery systems throughout the biomedical field. This journal is indexed on PubMed Central,

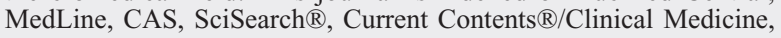

Journal Citation Reports/Science Edition, EMBase, Scopus and the Elsevier Bibliographic databases. The manuscript management system is completely online and includes a very quick and fair peer-review system, which is all easy to use. Visit http://www.dovepress.com/ testimonials.php to read real quotes from published authors.

Submit your manuscript here: http://www.dovepress.com/international-journal-of-nanomedicine-journal 